\title{
Reconsidering complete search algorithms for protein backbone NMR assignment
}

\author{
Olga Vitek ${ }^{1, *}$, Chris Bailey-Kellogg ${ }^{3}$, Bruce Craig ${ }^{1}$, Paul Kuliniewicz ${ }^{2}$ and Jan Vitek ${ }^{2}$ \\ ${ }^{1}$ Department of Statistics and ${ }^{2}$ Department of Computer Sciences, Purdue University, West Lafayette, \\ IN 47907, USA and ${ }^{3}$ Department of Computer Science, Dartmouth College, Hanover, NH 03755, USA
}

\begin{abstract}
Motivation: Nuclear magnetic resonance (NMR) spectroscopy is widely used to determine and analyze protein structures. An essential step in NMR studies is determining the backbone resonance assignment, which maps individual atoms to experimentally measured resonance frequencies. Performing assignment is challenging owing to the noise and ambiguity in NMR spectra. Although automated procedures have been investigated, by-and-large they are still struggling to gain acceptance because of inherent limits in scalability and/or unacceptable levels of assignment error. To have confidence in the results, an algorithm should be complete, i.e. able to identify all solutions consistent with the data, including all arbitrary configurations of extra and missing peaks. The ensuing combinatorial explosion in the space of possible assignments has led to the perception that complete search is hopelessly inefficient and cannot scale to realistic datasets.
\end{abstract}

Results: This paper presents a complete branch-contract-and-bound search algorithm for backbone resonance assignment. The algorithm controls the search space by hierarchically agglomerating partial assignments and employing statistically sound pruning criteria. It considers all solutions consistent with the data, and uniformly treats all combinations of extra and missing data. We demonstrate our approach on experimental data from five proteins ranging in size from 70 to 154 residues. The algorithm assigns $>95 \%$ of the positions with $>98 \%$ accuracy. We also present results on simulated data from 259 proteins from the RefDB database, ranging in size from 25 to 257 residues. The median computation time for these cases is $1 \mathrm{~min}$, and the assignment accuracy is $>99 \%$. These results demonstrate that complete search not only has the advantage of guaranteeing fair treatment of all feasible solutions, but is efficient enough to be employed effectively in practice.

Availability: The $\mathrm{MBA}_{2}$ software package is made available under an open-source software license. The datasets featured in the Results section can also be obtained from the contact author.

Contact: ovitek@stat.purdue.edu

\section{INTRODUCTION}

Nuclear magnetic resonance (NMR) spectroscopy is an experimental method capable of determining 3D structures of proteins in atomic detail under nearly physiological conditions. Some $15-20 \%$ of new protein structures are currently determined by NMR, and the rate is likely to grow (Montelione et al., 2000). One of the bottlenecks in NMR-based studies is backbone resonance assignment, a procedure

${ }^{*}$ To whom correspondence should be addressed. establishing values of the chemical shifts of the atoms of the protein backbone. Chemical shifts can be viewed as magnetic signatures of the atoms and are extensively used in analyses of structure, dynamics and molecular interactions.

Backbone resonance assignment typically uses a set of 3D NMR experiments. An example of such an experiment is the $\mathrm{HN}(\mathrm{CO}) \mathrm{CA}$ shown in Figure 1a, which magnetically correlates a bonded pair of $\mathrm{H}^{\mathrm{N}}-\mathrm{N}$ backbone nuclei with the $\mathrm{C}^{\alpha}$ nucleus of the preceding residue, and yields a 3D spectrum. Peaks in the spectrum indicate the triples of $\mathrm{H}^{\mathrm{N}}-\mathrm{N}-\mathrm{C}^{\alpha}$ nuclei that exhibit magnetic interactions. The coordinates of the peaks are the chemical shifts of the nuclei. Each $\mathrm{HN}(\mathrm{CO}) \mathrm{CA}$ peak records signals from two neighboring residues and, therefore, captures sequential interactions. Another 3D experiment, HNCA, magnetically correlates the bonded $\mathrm{H}^{\mathrm{N}}-\mathrm{N}$ pairs with the $\mathrm{C}^{\alpha}$, either of the preceding residue [as in the $\mathrm{HN}(\mathrm{CO}) \mathrm{CA}$ ] or of the same residue. It yields approximately twice as many 3D peaks as the $\mathrm{HN}(\mathrm{CO}) \mathrm{CA}$, gathering both sequential and within-residue magnetic interactions. Similar NMR experiments can be designed to correlate the $\mathrm{H}^{\mathrm{N}}-\mathrm{N}$ pairs with $\mathrm{C}^{\beta}, \mathrm{H}^{\alpha}$ and $\mathrm{C}^{\prime}$. Coordinates of peaks from the various experiments can be combined by reference to shared coordinates of the $\mathrm{H}^{\mathrm{N}}-\mathrm{N}$ resonance types. The resulting spin systems, shown in Figure 1b, contain chemical shifts of the anchor $\mathrm{H}^{\mathrm{N}}-\mathrm{N}$ nuclei, of other backbone nuclei within the same residue and of nuclei in the sequentially preceding residue.

Although NMR studies assume that the primary sequence is known, the spectra provide no information about which position in the sequence generated a particular chemical shift. This must be inferred from the observed spin systems by the process of backbone resonance assignment. A typical resonance assignment procedure (Moseley and Montelione, 1999) searches for mappings between spin systems and positions in the sequence that satisfy the following constraints (Fig. 1b): (1) For two spin systems mapped to adjacent positions, the within-residue chemical shifts of the first match the sequential chemical shifts of the second. (2) Each spin system mapped to a position is aligned with the amino acid type, meaning that its chemical shifts are consistent with the expected values for the amino acid. (3) Each spin system is mapped to at most one position in the protein sequence, and each position is mapped to at most one spin system.

Noise and ambiguity in the spectra reduce the effectiveness of these constraints in the resonance assignment process. First, peak coordinates are uncertain, so approximate matches in constraint (1) must be allowed within prespecified tolerance values. All matches must be allowed in the case of a missing chemical shift. Second, the ranges of chemical shifts for an amino acid type in constraint (2) are 
(a)

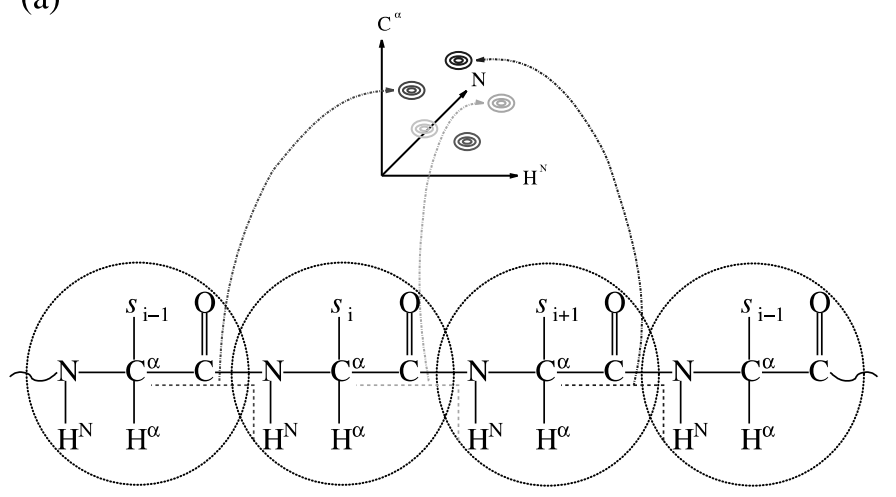

(b) $\mathbf{x}_{i 1}$
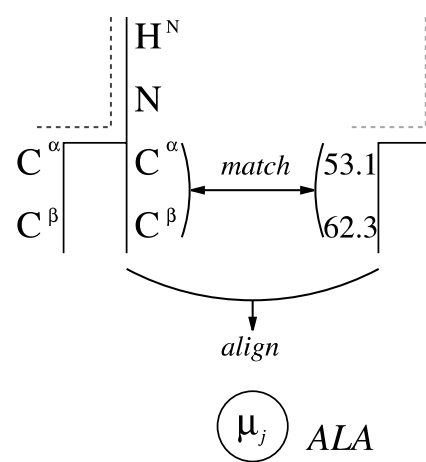

$A L A$
$\mathbf{X}_{i 2}$

8.31

112

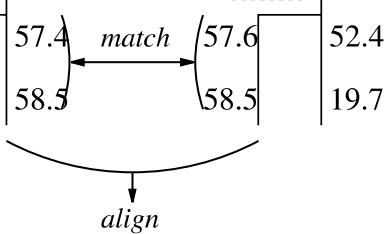

$\mu_{j+1}$

$A S P$

Fig. 1. NMR data. (a) The $\mathrm{HN}(\mathrm{CO}) \mathrm{CA}$ experiment correlates the $\mathrm{H}^{\mathrm{N}}-\mathrm{N}$ pair from one residue with the $\mathrm{C}^{\alpha}$ from the preceding residue. Spectral peaks capture various such interactions. Coordinates of the peaks are the chemical shifts of the involved atoms. (b) Compilation of peaks from a set of experiments yields spin systems $\mathbf{x}_{i}$ that represent chemical shifts of atoms within a residue and in the sequentially preceding residue. Resonance assignment algorithms proceed by matching the chemical shifts of pairs of spin systems and aligning them at adjacent positions in the primary sequence.

fairly broad, and usually allow multiple mappings of spin systems to a position. Third, positions in the sequence can have entirely missing spin systems, and the number and identity of such positions is unknown. At the same time, observed data can be extraneous.

These artifacts of noise and ambiguity result in a combinatorial explosion of the search space of candidate mappings. Consequently, exhaustive search algorithms (Andrec et al., 2001; Lin et al., 2002; Vitek et al., 2004) have been dismissed as impractical for anything but small proteins and 'clean' datasets. Semi-automated procedures such as CAMRA (Gronwald et al., 1998), MAPPER (Güntert et al., 2000) and PACES (Coggins and Zhou, 2003) require human intervention to manage the search space. Fully automated approaches gain in scalability but compromise accuracy or efficiency by using, e.g. bestfirst search (Zimmerman et al., 1997), approximation algorithms (Chen et al., 2003), and global and local stochastic optimization, as in, for example, the random graph approach (Bailey-Kellogg et al., 2004), MARS (Jung and Zweckstetter, 2004), MONTE (Hitchens et al., 2003) and TATAPRO (Atreya et al., 2000).

In order to fully characterize the confidence in an assignment, an algorithm must be complete. That is, it must be able to identify all solutions consistent with the data, including those with arbitrary configurations of matches and placements of extra and missing peaks. It is not sufficient to focus on optimization for just the 'best' solution, since the ranking may be sensitive to small details in the method used to evaluate the quality of the satisfaction of the constraints. However, it is not appropriate to treat all solutions as plausible, as statistical scoring models can provide estimates of quality and indicate that some solutions are clearly inconsistent with the data. Finally, it is dangerous to fix 'unambiguous' chains of matched spin systems. This does not appropriately represent our uncertainty regarding the process that generated the data, since a 'break' owing to an entirely missing spin system can, in principle, appear at any position.

We present here the first efficient algorithm that performs a complete search for backbone resonance assignment. It uniformly treats all matches and combinations of extra and missing data, and returns all assignments that are statistically consistent with the data. Our branch-contract-and-bound algorithm explores the space of admissible solutions, not a single solution at a time, but in groups of partial solutions, such that entire sets of infeasible solutions can be ruled out simultaneously. The algorithm branches on choices of restrictions on missing data and on selections of partial mappings, and prunes according to both local and global statistical criteria. Since missing data essentially act as wildcards, we explore all possible combinations of missing by gradually increasing their number until solutions are found. We employ a Bayesian probability model for NMR spectra (Vitek et al., 2004) which serves as a scoring function in the search for candidate mappings. This model appropriately assesses uncertainty, is amenable to formal statistical inference and contributes greatly to the high accuracy of our algorithm.

\section{METHODS}

\subsection{Scoring function}

Consider a primary sequence of $R$ residues. Let $\boldsymbol{\mu}=\left(\boldsymbol{\mu}_{1}, \ldots, \boldsymbol{\mu}_{R}\right)$ denote the unknown 'true' chemical shifts of the backbone nuclei of the protein. Here each $\boldsymbol{\mu}_{j}$ is a vector composed of individual chemical shifts $\mu_{t j}$ for each resonance type $t=1, \ldots, T$ at position $j$. The $\boldsymbol{\mu}_{j}$ are the parameters of interest, and the goal of the backbone resonance assignment is to estimate these values. The input data are $I$ observed spin systems $\mathbf{x}=\left\{\left(\mathbf{x}_{1}^{s}, \mathbf{x}_{1}^{w}\right), \ldots,\left(\mathbf{x}_{I}^{s}, \mathbf{x}_{I}^{w}\right)\right\}$, where $\mathbf{x}_{i}^{s}$ is the vector of sequential chemical shifts $x_{t i}^{s}$, and $\mathbf{x}_{i}^{w}$ is the vector of within-residue chemical shifts $x_{t i}^{w}$, over resonance type $t$. We assume that the spin systems are correctly and unambiguously compiled prior to the analysis. The total number of spin systems $I$ can be greater than, equal to or less than the length of the protein $R$, depending on the presence of extra and missing spin systems.

Let $\boldsymbol{a}=\left(a_{1}, \ldots, a_{R}\right)$ be a candidate mapping of the observed spin systems to positions in the primary sequence. Here $a_{j}=i$ if $\mathbf{x}_{i}^{w}$ is mapped to position $j$ (or equivalently $\mathbf{x}_{i}^{s}$ is mapped to position $j-1$ ). We will also use the notation $\boldsymbol{a}=(j, i)$ to emphasize the relationship between positions in the sequence and spin systems. A candidate mapping is one-to-one and gives the putative origin of the observed data. Some of the spin systems can be considered as extras by $\boldsymbol{a}$ and will be associated with sources of noise. According to the Bayesian paradigm, comparison between candidate mappings requires (1) specification of the probability distribution of the observed data $\mathbf{x}$; (2) specification of prior distributions of the unknown parameters; (3) integrating out the unknowns with respect to the prior distributions; and (4) calculation of posterior probabilities $\operatorname{Pr}(\boldsymbol{a} \mid \mathbf{x})$ by applying Bayes theorem. The remainder of this section details these steps.

2.1.1 Likelihoods We view the observed chemical shifts $\mathbf{x}$ as noisy readings from the unknown true chemical shifts $\boldsymbol{\mu}$. We assume that errors of the 
(a)

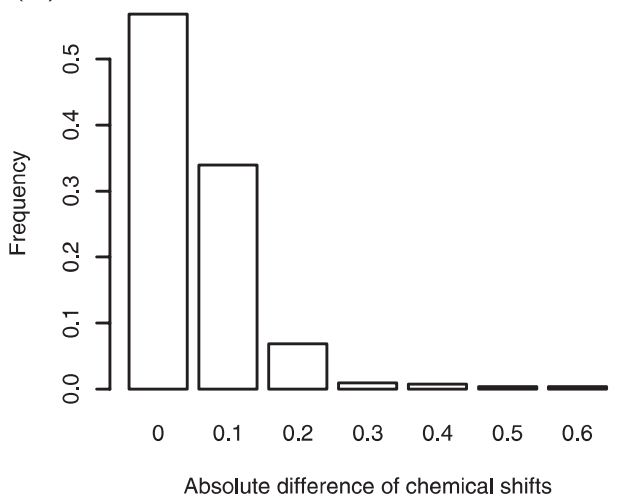

(b)

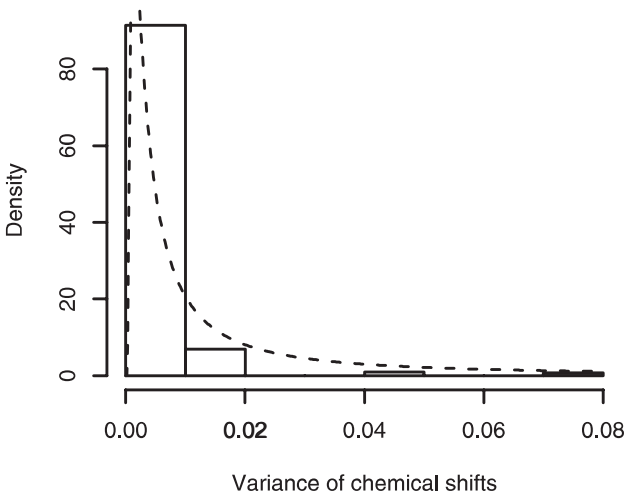

Fig. 2. (a) Histogram of absolute match differences of $\mathrm{C}^{\alpha}$ resonance type combined from six AutoAssign datasets. (b) Histogram of estimates of experimental variance obtained as a transformation of match differences. Dashed line is a fitted scaled inverse $\chi^{2}$-distribution with one degree of freedom.

readings are independent across positions and across resonance types, and are normally distributed. Specifically,

$$
\mathbf{x}_{a_{j}}^{s} \mid \boldsymbol{\mu}, \boldsymbol{V}, \boldsymbol{a} \sim \mathcal{N}\left(\boldsymbol{\mu}_{j-1}, V_{j-1}\right) \quad \text { and } \quad \mathbf{x}_{a_{j}}^{w} \mid \boldsymbol{\mu}, \boldsymbol{V}, \boldsymbol{a} \sim \mathcal{N}\left(\boldsymbol{\mu}_{j}, V_{j}\right) .
$$

Matrices $V_{j}$ are unknown experimental variances that need not be identical for all $j$. Independence across resonance types implies that $V_{j}$ are diagonal, and we denote the non-zero elements as $v_{t j}^{2}$.

2.1.2 Priors for $\boldsymbol{\mu}, \boldsymbol{V}$ and $\boldsymbol{a}$ A prior distribution for the chemical shifts $\boldsymbol{\mu}_{j}$ has been proposed by Marin et al. (2004), and is a result of a comprehensive study of entries in the database BioMagResBank (Seavey et al., 1991, http://www.bmrb.wisc.edu). The distribution is residue-type specific and takes into account the overrepresentation of certain protein sequences in the database as well as the correlation of chemical shifts within a residue type. Formally, $\boldsymbol{\mu}_{j} \sim \mathcal{N}\left(\boldsymbol{\theta}_{j}, \Sigma_{j}\right)$, where $\boldsymbol{\theta}_{j}$ is a known vector, and $\Sigma_{j}$ is a known non-diagonal matrix.

The prior distribution of $V_{j}$ relaxes the stringent assumption of constant and known experimental variances made in our previous work (Vitek et al., 2004). It is obtained by examining the estimates of experimental variances for each resonance type, namely $\frac{1}{2}\left(x_{t\left(a_{j+1}\right)}^{s}-x_{t\left(a_{j}\right)}^{w}\right)^{2}$, in six datasets provided as a test to the AutoAssign program (Zimmerman et al., 1997). As shown in Figure 2 for the case of $\mathrm{C}^{\alpha}$, we fit the histograms with scaled inverse $\chi^{2}$-distributions with one degree of freedom having densities

$$
f\left(v_{t j}^{2}\right)=\frac{1}{\sqrt{2 \pi} v_{t j}^{3}} \exp \left(-\frac{S_{t}^{2}}{2 v_{t j}^{2}}\right) .
$$

The scale parameters $S_{t}^{2}$ depend on the resonance type, and the specific values are 0.0016 p.p.m. ${ }^{2}$ for $\mathrm{C}^{\prime}, 0.004$ p.p.m. ${ }^{2}$ for $\mathrm{C}^{\alpha}, 0.005$ p.p.m. ${ }^{2}$ for $\mathrm{C}^{\beta}$ and 0.00005 p.p.m. ${ }^{2}$ for $\mathrm{H}^{\alpha}$. The choice of one degree of freedom comes from the fact that all experimented variances are estimated on the basis of two data points.

The prior distribution of mappings $\boldsymbol{a}$ is used to determine the number of entirely missing spin systems (or, equivalently, the number of extra spin systems) in the dataset. By analogy with the model selection literature, we use Bayesian information criterion (BIC) weights to penalize mappings with an excessive number of extra spin systems. Specifically, $\log \operatorname{Pr}(\boldsymbol{a}) \propto(\log N) \cdot R^{\prime}$ for mappings where $N$ is the total number of observed chemical shifts, and $R^{\prime}$ is the number of chemical shifts considered as noise. Furthermore, we assume a uniform prior distribution of missing spin systems in the sequence conditionally on their total number. This appropriately represents our uncertainty in the physical process producing missing spin systems, but requires a search algorithm considering the possibility of a missing spin system at each position in the sequence.

2.1.3 Marginal likelihoods The marginal likelihood $\operatorname{Pr}(\mathbf{x} \mid \boldsymbol{a})$ of the data given a mapping can be obtained by integrating out the unknown $\boldsymbol{\mu}_{j}$ and $V_{j}$ with respect to their prior distributions. In our case,

$$
\begin{aligned}
\operatorname{Pr}(\mathbf{x} \mid \boldsymbol{a}) \approx & \prod_{j=1}^{R} \prod_{t=1}^{T} \mathcal{C}\left[\frac{1}{\sqrt{2} S_{t}}\left(x_{t\left(a_{j+1}\right)}^{s}-x_{t\left(a_{j}\right)}^{w}\right)\right] \\
& \times \prod_{j=1}^{R} \phi\left[\Sigma_{j}^{-1 / 2}\left(\overline{\mathbf{x}}_{a_{j}}-\boldsymbol{\theta}_{j}\right)\right]
\end{aligned}
$$

where $\mathcal{C}$ denotes the density of the standard Cauchy distribution, $\phi$ denotes the density of the standard multivariate normal distribution and $\overline{\mathbf{x}}_{a_{j}}$ is the average of $\mathbf{x}_{a_{j+1}}^{s}$ and $\mathbf{x}_{a_{j}}^{w}$. The first term in Equation (1) evaluates the plausibility of the match at a position $j$. As shown in in Figure 3 a for the case of $\mathrm{C}^{\alpha}$, it gives more weight to tight matches than other scoring functions, but has heavier tails. The second term in Equation (1) evaluates the plausibility of the alignment. As shown in in Figure $3 b$ for the case of $C^{\alpha}$ and $C^{\beta}$ for alanine, it takes into account both the range and the correlation of the chemical shifts.

2.1.4 Posterior probabilities Through the application of the Bayes theorem, $\operatorname{Pr}(\boldsymbol{a} \mid \mathbf{x}) \propto \operatorname{Pr}(\mathbf{x} \mid \boldsymbol{a}) \cdot \operatorname{Pr}(\boldsymbol{a})$. The posterior probabilities are scores used to compare the candidate mappings.

\subsection{Algorithm for complete search}

We develop here a complete, fully automated, backbone resonance assignment algorithm. Our algorithm explores the space of all plausible assignments with a branch-and-bound (more precisely, branch-contract-and-bound) search technique, illustrated in Figure 4. Nodes in a search tree compactly represent partial assignment solutions. The search recursively expands 'promising' nodes, eventually identifying entire assignments at the leaves. The expansion of a node branches on the possible placements for individual spin systems, as well as constraints on the number of missings. Expanded nodes are evaluated and pruned according to bounds that test consistency with the data according to the statistical scoring criteria, as well as plausibility relative to other solutions. In order to enhance the effectiveness of the bounds, we employ an additional contraction step between branching and bounding. Contraction takes advantage of the reduced combinatorics in the context of a branch, and generates combinations of partial solutions to be tested by the bound.

The practical utility of branch-and-bound algorithms critically depends on the effectiveness of the branching and bounding steps, and naïve approaches generally do not scale. Below we discuss the particular insights underlying our approach which result in the first complete algorithm able to handle large and noisy proteins.

Let us define a strand $D=\left\langle\left(j, i_{1}\right), \ldots,\left(j+|D|, i_{|D|}\right)\right\rangle$ as a partial assignment of spin systems $i$ to consecutive positions $j$. Then let a window, $W=\left\{D_{1}, \ldots, D_{|W|}\right\}$, be a set of alternative strands covering the same 
(a)

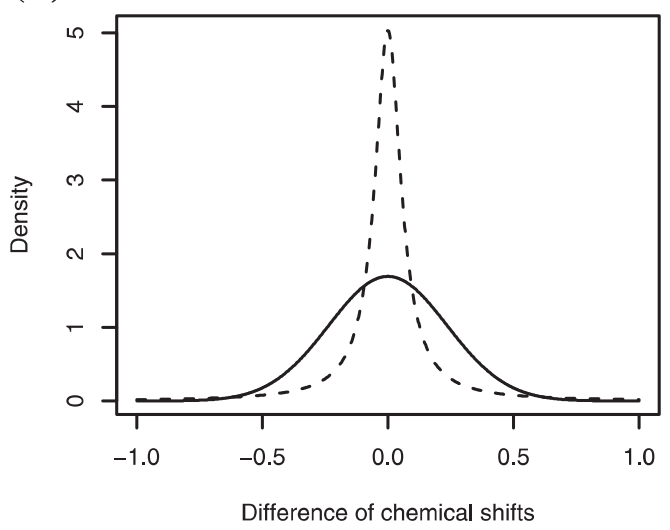

(b)

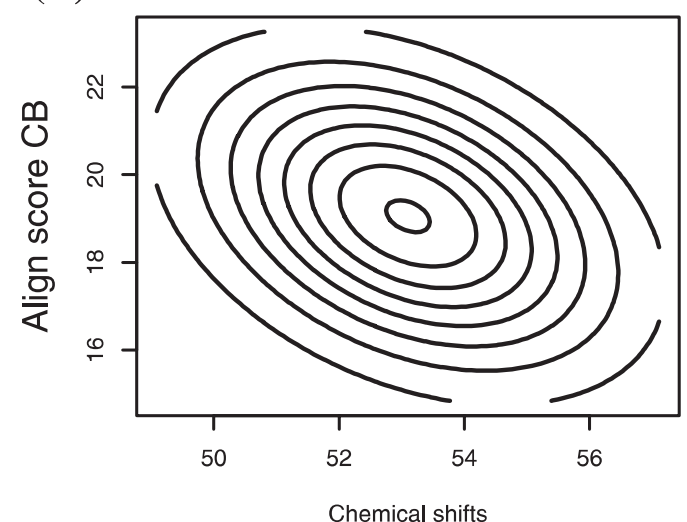

Fig. 3. (a) Plausibility of a match between two $C^{\alpha}$ chemical shifts. Dashed line: Scaled Cauchy density in Equation (1). Solid line: A normal density where the standard deviation is equal to one-third of the standard match tolerance of the $\mathrm{C}^{\alpha}$ resonance type, namely $0.5 / 3=0.17$. The latter is used by resonance assignment methods, such as MARS. (b) Plausibility of aligning $\mathrm{C}^{\alpha}$ and $\mathrm{C}^{\beta}$ chemical shifts to an alanine residue. Solid lines are level curves of the multivariate normal distribution in Equation (1).

positions but with different combinations of spin systems. With these definitions, the search space $\mathcal{S}=\left\{W_{1}, \ldots, W_{|\mathcal{S}|}\right\}$ can be represented as a set of disjoint windows that cover all residues. A candidate mapping $\boldsymbol{a}$ has one strand selected for each window. We maintain a set $\mathcal{A}=\left\{\boldsymbol{a}_{1}, \ldots, \boldsymbol{a}_{|\mathcal{A}|}\right\}$ of candidate mappings discovered during the course of the search, and explore the search tree as follows. We also maintain the mapping $\boldsymbol{a}^{*}$ with the highest posterior probability found so far.

2.2.1 Initialization For a protein with $R$ residues and $I$ spin systems, we initialize the search space $\mathcal{S}=\left\{W_{1}, \ldots, W_{R}\right\}$ with $R$ windows. Each window $W_{j}=\{(j, 1),(j, 2), \ldots,(j, I),(j, I+1)\}$ contains $I+1$ unit-length strands, one for each spin system and one for a 'wildcard' representing a missing spin system.

2.2.2 Branching The search space is split according to two types of branches: a 'missing' branch and a 'mapping' branch. A missing branch places an upper bound on the number of missing placeholders that can be used in a window. Only strands with at most the specified number of missings are considered in the subtree. Thus different missing branches for the same window explore solutions that leave different numbers of positions as wildcards. A mapping branch fixes a spin system at a position in the sequence.

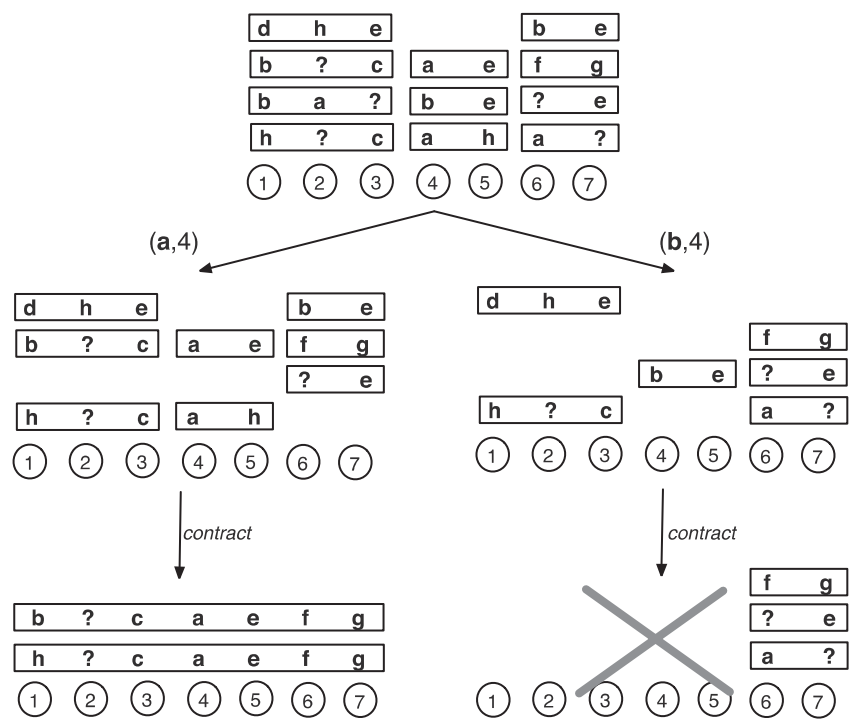

Fig. 4. Branch-contract-and-bound algorithm. The topmost node consists of three windows covering, respectively, positions (1-3), (4-5) and (6-7) with, respectively 4,3 and 4 different strands. Letters a-h denote spin systems, and ? entirely missing spin systems. The left-hand branches fixes a at position 4 . As a result, the space is pruned of strands that conflict with this assignment. The contract step yields two complete mappings (b?caefg) and (h?caefg) which differ only in one position. The right-hand branch fixes $b$ at 4 , and after the contraction, we find no consistent mappings. This branch is, thus, a dead end.

Strands in the subtree are filtered according to the mapping, so that the same spin system is not mapped to multiple locations, and multiple spin systems are not mapped to the same location. Different mapping branches thus explore different hypotheses about individual position-spin system mappings.

2.2.3 Leaf nodes The expansion of a node ends when it is identified as either a leaf node or a dead end. A leaf node has been reached when the space $\mathcal{S}=\{W\}$ has a single window $W$ covering the entire protein. In this case, all strands in $W$ are added to the set of candidate mappings $\mathcal{A}$, and the best mapping $\boldsymbol{a}^{*}$ is updated accordingly. A dead end occurs when the space $\mathcal{S}=\left\{W_{1}, \ldots, W_{|\mathcal{S}|}\right\}$ has some window $W_{k}=\emptyset$. That is, no combination of spin systems can be mapped to the positions of the window with respect to the branching constraints, and thus there is no need to explore further. In both cases the algorithm backtracks and considers the next alternative branch.

2.2.4 Contraction After each branch, the algorithm contracts the search space by merging adjacent windows. A pair of windows $W$ and $W^{\prime}$ covering positions $\{j, \ldots, k\}$ and $\{k+1, \ldots, l\}$ is merged into a new window covering positions $\{j, \ldots, l\}$. It contains a subset of the strands $\left\{D \cup D^{\prime} \mid D \in W, D^{\prime} \in W^{\prime}\right\}$ that are consistent with the bound. If the number of strands in the new window does not exceed a user-specified threshold, we substitute it for the original two windows; otherwise, we leave the original windows. We then iterate, until no adjacent windows can be merged.

2.2.5 Bounding Properties of the NMR spectra, along with the probability model in the previous section, can be used to bound the search space. Specifically, we evaluate the quality of each strand at a node in the tree, and determine whether the strand should be kept. Bounds (1)-(3) arise from the constraints in Section 1, and from the functional form of Equation (1):

(1) Match: $\left|x_{t, a_{j+1}}^{s}-x_{t, a_{j}}^{w}\right| \leq \xi_{t}$ for all $t, j$ and $\boldsymbol{a}$. Here $\xi_{t}$ are typical match tolerances in NMR studies, namely 0.25 p.p.m. for $\mathrm{C}^{\prime}, 0.5$ p.p.m. for $\mathrm{C}^{\alpha}$ and $\mathrm{C}^{\beta}$, and 0.05 p.p.m. for $\mathrm{H}^{\alpha}$. $\xi_{t}$ determines the valid chemical shift differences as in Figure 3a. 
(2) Align: $\Sigma_{j}^{-1 / 2}\left(\overline{\mathbf{x}}_{a_{j}}-\boldsymbol{\theta}_{j}\right) \leq$ quantile $\left(\chi_{T}^{2}, 0.9999\right)$ for all $j$ and $\boldsymbol{a}$. The quantile determines a level curve, such as in Figure $3 \mathrm{~b}$, which encircles the valid region of chemical shifts for the residue at position $j$.

(3) Unique map: If $a_{j}$ is not a missing spin system, then $a_{j} \neq a\left(j^{\prime}\right)$ for all $j$ and $j^{\prime}$.

(4) and (5) are global bounds derived from the probability model, based on the solutions discovered so far.

(4) Posterior probability: $P\left(\mathbf{x} \mid \boldsymbol{a}^{*}\right) / P(\mathbf{x} \mid \boldsymbol{a}) \leq 100$ for all $\boldsymbol{a}$. In other words, we need not consider mappings that are $>100$ times less likely a posteriori than $\boldsymbol{a}^{*}$.

(5) Number of missings: $\operatorname{Miss}(\boldsymbol{a}) \leq \operatorname{Miss}\left(\boldsymbol{a}^{*}\right)+1$ for all $\boldsymbol{a}$. The prior distribution of candidate mappings heavily penalizes additional missing spin systems. In the vast majority of cases it is sufficient to consider mappings where the number of missing spin systems does not exceed the number of missing spin systems in $\boldsymbol{a}^{*}$ by $>1$.

2.2.6 Heuristics When faced with choices of branches in the tree, we must decide which branch to explore first. In practice, we partition the branches to perform all missing branches first, followed by mapping branches for the rest of the tree. The policy for missing branches is to monotonically increase the number of missings. When taking a mapping branch, it is necessary to decide which position to fix and in which order to try assigning spin systems. Experimental results suggest that an effective policy is to always select the position with the smallest set of alternative spin systems that could be mapped to it. Once a position has been selected, we order the alternative spin systems according to the the likelihood of the strands in which they occur. This policy tends to reduce the width of the tree and typically find solutions significantly faster than other policies. Finally, in choosing which windows to contract first, we prefer windows with fewer strands, as they are less likely to cause a combinatorial explosion.

\subsection{Interpreting the results}

The probability model above can be used to make inference regarding mappings of the observed spin systems to positions. In the following, we say that a position has a reliable mapping if it is mapped to the same spin system according to all solutions satisfying bounds (1)-(5).

The probability model can also be used for inference regarding the unknown chemical shifts. Given a candidate mapping $\boldsymbol{a}$, the posterior distribution of a chemical shift $\mu_{t j}$ is a scaled Cauchy distribution truncated by the match tolerances $\xi_{t}$. The overall posterior distribution of $\mu_{t j}$ can be obtained by averaging over the candidate mappings $\boldsymbol{a}_{k}$ :

$$
\operatorname{Pr}\left(\mu_{t j} \mid \mathbf{x}\right)=\sum_{k=1}^{K} \operatorname{Pr}\left(\mu_{t j} \mid \mathbf{x}, \boldsymbol{a}_{k}\right) \operatorname{Pr}\left(\boldsymbol{a}_{k} \mid \mathbf{x}\right)
$$

In the following, we say that a chemical shift $\mu_{t j}$ is reliably determined if its posterior variance is within the range of variances for the resonance type $t$. We say that it is correctly determined if the difference between the posterior mean of $\mu_{t j}$ and the reference value does not exceed $\xi_{t}$.

\section{RESULTS}

\subsection{Experimental datasets}

We use our algorithm to analyze several publicly available experimental datasets: Human Ubiquitin (UCL/LICR, 2005, http://www.biochem.ucl.ac.uk/bsm/nmr/ubq/), and Zdomain, CspA, Ns1, RnaseWt, RnaseC6572S and Fgf (Zimmerman et al., 1997). Figure 5 describes the datasets and the corresponding reference solutions. The length of the proteins is between small and average for modern NMR studies.

As shown in Figure 6, execution completed in $<3 \mathrm{~h}$ for five proteins. Multiple mappings were found in all cases. The agreement between reliably mapped spin systems and the reference solution is

\begin{tabular}{|c|c|c|c|c|c|}
\hline \multirow[t]{2}{*}{ Protein } & \multirow[t]{2}{*}{ Length } & \multirow{2}{*}{$\begin{array}{c}\text { Miss } \\
\text { ss }\end{array}$} & \multirow{2}{*}{$\begin{array}{c}\text { Extra } \\
\text { ss }\end{array}$} & \multicolumn{2}{|c|}{ Resonance types } \\
\hline & & & & Sequential & Within \\
\hline Ubiquitin & 76 & 2 & 0 & $\mathrm{C}^{\prime}, \mathrm{C}^{\alpha}, \mathrm{C}^{\beta}$ & $\mathrm{C}^{\prime}, \mathrm{C}^{\alpha}, \mathrm{C}^{\beta}$ \\
\hline Zdomain & 70 & 2 & 2 & $\mathrm{C}^{\prime}, \mathrm{C}^{\alpha}, \mathrm{C}^{\beta}, \mathrm{H}^{\alpha}$ & $\mathrm{C}^{\alpha}, \mathrm{C}^{\beta}, \mathrm{H}^{\alpha}$ \\
\hline CspA & 70 & 2 & 4 & $\mathrm{C}^{\prime}, \mathrm{C}^{\alpha}, \mathrm{C}^{\beta}, \mathrm{H}^{\alpha}$ & $\mathrm{C}^{\alpha}, \mathrm{C}^{\beta}, \mathrm{H}^{\alpha}$ \\
\hline Ns1 & 73 & 4 & 2 & $\mathrm{C}^{\prime}, \mathrm{C}^{\alpha}, \mathrm{C}^{\beta}, \mathrm{H}^{\alpha}$ & $\mathrm{C}^{\alpha}, \mathrm{C}^{\beta}, \mathrm{H}^{\alpha}$ \\
\hline RnaseW & 124 & 0 & 37 & $\mathrm{C}^{\prime}, \mathrm{C}^{\alpha}, \mathrm{C}^{\beta}, \mathrm{H}^{\alpha}$ & $\mathrm{C}^{\prime}, \mathrm{C}^{\alpha}, \mathrm{C}^{\beta}, \mathrm{H}^{\alpha}$ \\
\hline RnaseC & 124 & 0 & 37 & $\mathrm{C}^{\prime}, \mathrm{C}^{\alpha}, \mathrm{C}^{\beta}, \mathrm{H}^{\alpha}$ & $\mathrm{C}^{\prime}, \mathrm{C}^{\alpha}, \mathrm{C}^{\beta}, \mathrm{H}^{\alpha}$ \\
\hline Fgf & 154 & 2 & 24 & $\mathrm{C}^{\prime}, \mathrm{C}^{\alpha}, \mathrm{C}^{\beta}, \mathrm{H}^{\alpha}$ & $\mathrm{C}^{\prime}, \mathrm{C}^{\alpha}, \mathrm{C}^{\beta}, \mathrm{H}^{\alpha}$ \\
\hline
\end{tabular}

Fig. 5. Description of the experimental datasets and reference solutions.

\begin{tabular}{|l||c|c|c|c|c|c|c|}
\hline Protein & $|\mathcal{A}|$ & Miss & $\mathbf{r}$ & $\mathbf{c}$ & $\mathbf{r}_{\mathrm{C}^{\alpha}}$ & $\mathbf{c}_{\mathrm{C}^{\alpha}}$ & Hours \\
\hline \hline Ubiquitin & 2 & 5 & 66 & $100 \%$ & 71 & $100 \%$ & 0.005 \\
\hline Zdomain & 3 & 3 & 62 & $98 \%$ & 68 & $100 \%$ & 0.02 \\
\hline CspA & 11 & 3 & 59 & $98 \%$ & 64 & $100 \%$ & 0.26 \\
\hline RnaseC & 2 & 1 & 118 & $100 \%$ & 124 & $100 \%$ & 2.44 \\
\hline Fgf & 4 & 4 & 140 & $99 \%$ & 151 & $100 \%$ & 0.74 \\
\hline
\end{tabular}

Fig. 6. Summary of assignment results for the experimental datasets. $|\mathcal{A}|$ is the number of candidate mappings satisfying bounds (1)-(5) in Section 2. Miss is the number of missing spin systems in the mapping with the highest posterior probability. The terms in the next four columns are introduced in Section 2: $\mathbf{r}$ is the number of positions with unambiguous mappings of spin systems, and $\mathbf{c}$ is the fraction of correct mappings among the reliable positions. $\mathbf{r}_{\mathrm{C}^{\alpha}}$ is the number of reliably determined chemical shifts, and $\mathbf{c}_{\mathrm{C}^{\alpha}}$ is the fraction of correctly determine chemical shifts among the reliable ones. Hours is the execution time in hours on a $2.5 \mathrm{GHz}$ PowerPC G5 with $3 \mathrm{~GB}$ of memory.

\begin{tabular}{|l|c|c|c|c|c|}
\hline Protein & \multirow{2}{*}{ Adjacency } & \multicolumn{2}{|c|}{ Free } & \multicolumn{2}{c|}{ Enforced } \\
\cline { 3 - 6 } & & $\mathbf{r}_{\mathrm{C}^{\alpha}}$ & $\mathbf{c}_{\mathrm{C}^{\alpha}}$ & $\mathbf{r}_{\mathrm{C}^{\alpha}}$ & $\mathbf{c}_{\mathrm{C}^{\alpha}}$ \\
\hline \hline RnaseC & $\mathbf{0 . 8 1}$ & 124 & $100 \%$ & 121 & $100 \%$ \\
\hline Zdomain & $\mathbf{0 . 5 4}$ & 68 & $100 \%$ & 71 & $95 \%$ \\
\hline Ubiquitin & $\mathbf{0 . 5 0}$ & 71 & $100 \%$ & 70 & $100 \%$ \\
\hline Fgf & $\mathbf{0 . 3 4}$ & 151 & $100 \%$ & 152 & $100 \%$ \\
\hline CspA & $\mathbf{0 . 0 0}$ & 64 & $100 \%$ & - & - \\
\hline
\end{tabular}

Fig. 7. Assignments with adjacency information. Adjacency is the proportion of the spin systems forming unambiguous chains. Free denotes the approach considering a missing spin system at each position in the sequence, and Enforced is the approach that enforces unambiguous matches. $\mathbf{c}_{\mathrm{C}^{\alpha}}$ and $\mathbf{r}_{C^{\alpha}}$ are as in Figure 6. Since CspA has 0 adjacency, one cannot enforce the connectivity information for this protein.

very good, but not perfect. This is attributable to match differences in the reference solutions that exceed the tolerances in bound (1) of Section 2. In such cases the algorithm typically introduces a missing spin system that compensates for the invalid match. Therefore, the number of missing spin systems is larger than the corresponding number in the reference solution. However, disagreements in the mappings of spin systems do not affect the inference regarding the chemical shifts-Figure 6 shows perfect agreement between the determined chemical shifts and the reference solution.

\subsection{Importance of missing spin systems in the search space}

Many existing algorithms for resonance assignment reduce the search space by compiling chains of unambiguously connected spin systems 
CLEAN
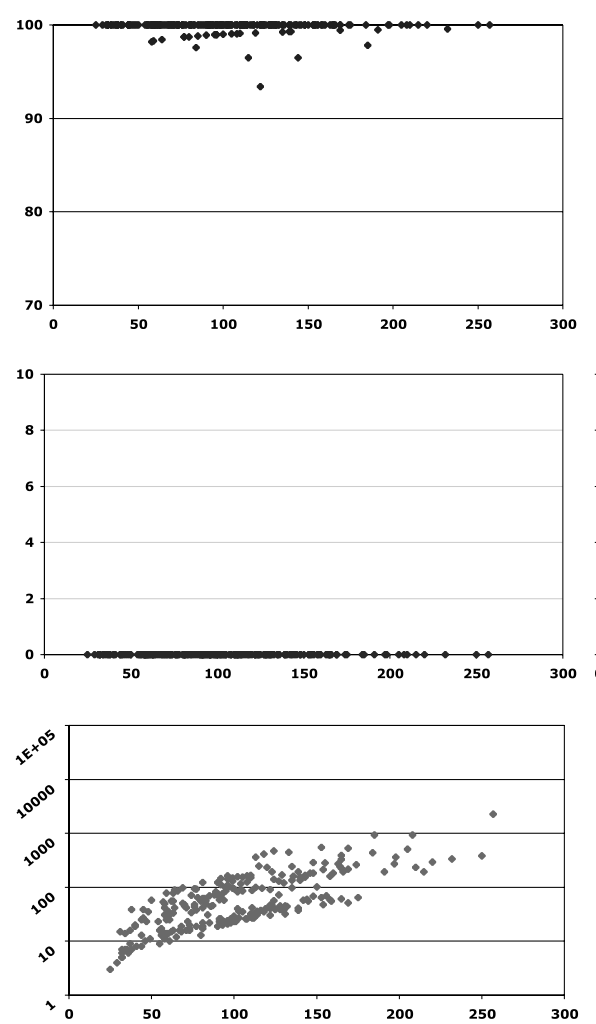

CONSISTENT
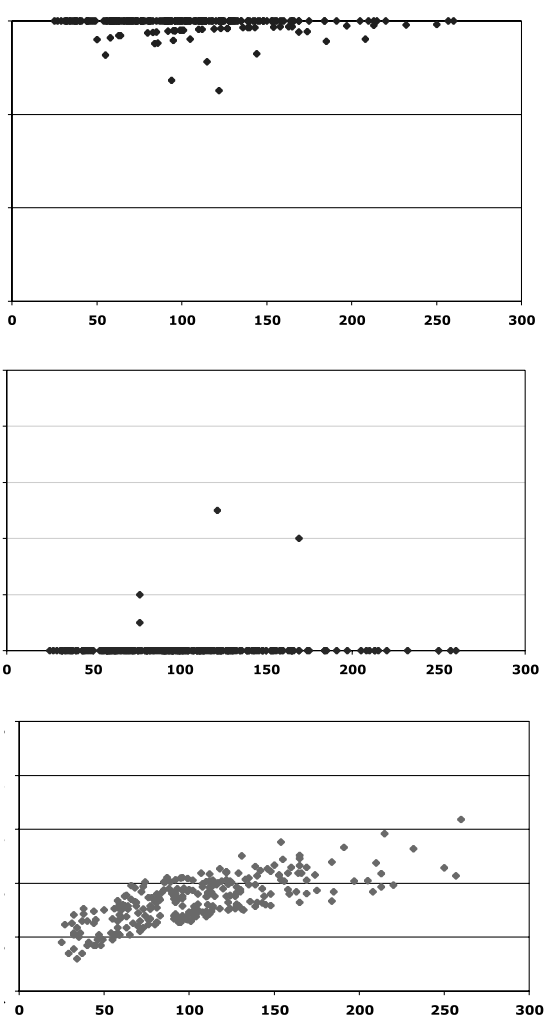

NOISY
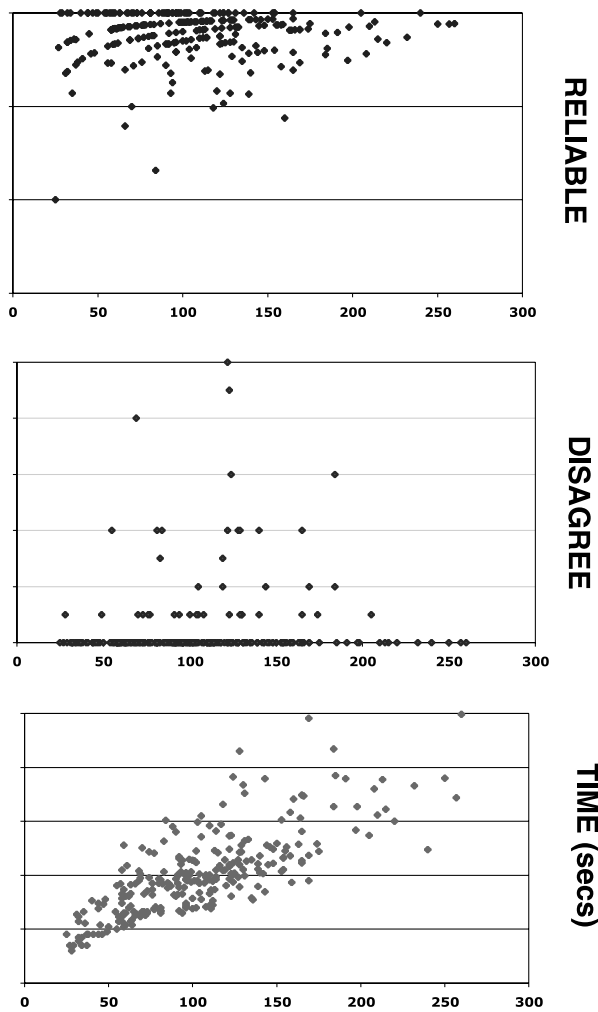

Fig. 8. Assignment results for 259 proteins selected randomly from RefDB. Clean datasets have spin systems with no experimental noise. Consistent datasets have distributions of experimental noise observed in the AutoAssign data, but within standard match tolerances. Noisy datasets have non-truncated experimental noise observed in the AutoAssign data. The $x$-axis is protein length (ranging between 25 and 257). The $y$-axes have the following interpretations. For Reliable, it shows the percentage of reliably mapped positions ( $r$ / number of assignable positions). For Disagree, it gives the absolute number of disagreements between reliably mapped spin systems and the reference solution. Finally, for Time, it shows computation time in seconds on a log scale. The median and maximum times are Clean: median $=0.7 \mathrm{~min}, \max =20 \mathrm{~min}$; consistent: median $=0.7 \mathrm{~min}, \max =25 \min$; noisy: median $=1.4 \mathrm{~min}, \max =27 \mathrm{~h}$.

with no breaks. In our opinion, ignoring the possibility of a 'break' at each position in the sequence underestimates the impact of missing spin systems, and of match differences that exceed the tolerance values. This section illustrates that point by comparing two approaches with resonance assignment. The first, proposed in this paper and called free in Figure 7, considers the possibility of a missing spin system at each position in the sequence. The second approach, proposed by Lin et al. (2002) and Chen et al. (2003), enforces unambiguous matches between spin systems. Specifically, if a spin system $\mathbf{x}_{1}$ can be uniquely followed by a spin system $\mathbf{x}_{2}$, and $\mathbf{x}_{2}$ can be uniquely preceded by $\mathbf{x}_{1}$, the match is considered as fixed. Therefore, matches between $\mathbf{x}_{1}$ and a missing, or between a missing and $\mathbf{x}_{2}$ are not allowed in the search space. The percentage of spin systems forming such unambiguous chains is a metric of 'density' of adjacency information.

The second column of Figure 7 describes the adjacency information in Human Ubiquitin and in the AutoAssign proteins. As can be seen, it ranges between 0 and 0.81 , suggesting that these data have less adjacency than the datasets in Chen et al. (2003), where the metric varies between 0.5 and 0.9. Furthermore, a comparison of Figure 6 and 7 suggests that adjacency is not a good measure of the difficulty of a dataset. Although CspA has adjacency information of 0 and RnaseC6572S has adjacency information of 0.8 , it takes almost 10 times longer to complete the assignment of RnaseC6572S.

According to columns 2-4 in Figure 7, enforcing adjacency in RnaseC6572S and Ubiquitin reduces the number of unambiguously mapped positions without compromising the accuracy of the determined $\mathrm{C}^{\alpha}$ chemical shifts. In Zdomain, it increases the number of unambiguously mapped positions at the expense of accuracy. Although these differences are small, they can be easily exacerbated in larger and noisier datasets.

\subsection{Synthetic data}

We evaluate the large-scale performance of our approach using synthetic data sets from 259 randomly selected entries to the database RefDB (Zhang et al., 2003). In order to generate datasets of realistic quality and size, we examined noise characteristics in the AutoAssign proteins, and simulated data according to these characteristics as follows. First, since it is unlikely that all the unreported chemical shifts in the database correspond to truly missing peaks, we simulate the missing values in the RefDB entries from the prior distributions (Marin et al., 2004), and compile spin systems on the basis of the full sets of chemical shifts. Second, we delete a random number of the correct spin systems, and add a random number of extra 
spin systems to the datasets. The two numbers were generated from Poisson distributions with mean 2 . Third, we randomly delete chemical shifts with frequencies observed in the AutoAssign proteins for each resonance type. Finally, we consider three scenarios of experimental noise. First, called clean, is unrealistic but often used to evaluate assignment procedures Jung and Zweckstetter (2004). It considers spin systems with no experimental noise. Second, called consistent, is realistic. Here, noise added to the chemical shifts is sampled from the histograms of match tolerances in the AutoAssign proteins, such as in Figure 2a. The histograms are truncated to satisfy the standard match tolerances for each resonance type. Third, called noisy, investigates the robustness of the proposed approach. It allows invalid match tolerances in the reference solution by sampling noise from the full (non-truncated) distribution of match differences in the AutoAssign proteins. In total, the procedure generated 777 synthetic datasets that are available from the contact author along with automated scripts for their execution.

The datasets were analyzed using the $\mathrm{MBA}_{2}$ software with default settings and no manual intervention. The results of the executions are summarized in Figure 8. As can be seen, the proposed procedure reliably determines most of the mappings between spin systems and positions. On average, $99.8 \%$ of assignable positions are unambiguously mapped in the clean datasets, and $99.7 \%$ of assignable positions are mapped in the noisy experiments. The accuracy of assignment is measured by $\mathbf{c}$, the proportion of reliable assignment that agree with the reference solution. It is on average $100 \%$ for clean data, $99.96 \%$ for consistent data and $99.36 \%$ for noisy data.

The last row in Figure 8 gives the execution times on a log scale. As expected, the execution time grows exponentially, in particular, in the noisy dataset where large numbers of missing spin systems are required to compensate for invalid matches. At the same time, the execution times remain within acceptable limits for the majority of the datasets.

\section{DISCUSSION}

We have presented the first algorithm for backbone resonance assignment that is complete and has been demonstrated to scale to datasets of realistic quality and size. We have validated our claims by conducting a large-scale automated resonance assignment study. The results show that, contrary to commonly accepted wisdom, complete search algorithm can handle problems of practical interest.

Where applicable, our approach will increase the number of reliably determined chemical shifts and yield fewer errors, as compared with existing methods. The characteristics that contribute to the excellent overall performance are (1) a rigorous probability model that assesses the uncertainty in the NMR spectra and is amenable to formal statistical inference; (2) a correct definition of the search space that considers the possibility of a missing spin system at any position in the sequence, and an arbitrary number of such missings; and (3) a complete algorithm that relies on hierarchical association of partial mappings to control the search space.

Complete algorithms are not a panacea. In our experimental study we encountered two AutoAssign datasets, Ns1 and RnaseWt, which proved challenging to the algorithm. But this is hardly surprising considering the quality of the data. Ns1 has four missing spin systems (out of 73 positions) and an average of $23 \%$ of the resonances missing per spin system. RnaseWt is missing $11 \%$ of its resonances and has 37 extraneous spin systems. Moreover, in both cases the reference solutions have a number of spin systems with scores outside the standard match tolerances (7 for RnaseWt and 5 for Ns1).

Complete search algorithms can, thus, be a very useful tool in many, if not all, situations. In cases of very noisy data, they can be used to guide heuristic or stochastic search algorithms that consider only promising portions of the space, sacrificing completeness for scalability.

\section{ACKNOWLEDGEMENTS}

We would like to thank Drs Gaetano Montelione and Hunter Moseley of the Center for Advanced Biotechnology and Medicine, Rutgers University, for providing access to the peak list files and the AutoPeak and AutoAssign programs.

Conflict of Interest: none declared.

\section{REFERENCES}

Andrec,M. et al. (2001) Protein structural motif recognition via NMR residual dipolar couplings. J. Am. Chem. Soc., 123, 1222.

Atreya,H.S. et al. (2000) A tracked approach for automated NMR assignments in proteins (TATAPRO). J. Biomol. NMR, 17, 125-136.

Bailey-Kellogg,C., Chainraj,S. and Pandurangan,G. (2004) A random graph approach to NMR sequential assignment. In Proceedings of The International Conference on Computational Molecular Biology (RECOMB), San Diego, California, USA, pp. 58-67.

Chen,Z.-Z. et al. (2003) Approximation algorithms for NMR spectral peak assignment. Theoretical Computer Science, 299, 211-229.

Coggins,B.E. and Zhou,P. (2003) PACES: Protein sequential assignment by computeraided exhaustive search. J. Biomol. NMR, 26, 93-111.

Gronwald,W. et al. (1998) CAMRA: Chemical shift based computer aided protein NMR assignments. J. Biomol. NMR, 12, 395-405.

Güntert,P. et al. (2000) Sequence-specific NMR assignment of proteins by global fragment mapping with program Mapper. J. Biomol. NMR, 17, 129-137.

Hitchens,T.K. et al. (2003) MONTE: An automated Monte Carlo based approach to nuclear magnetic resonance assignment of proteins. J. Biomol. NMR, 25, 1-9.

Jung,J. and Zweckstetter,M. (2004) MARS - robust automatic backbone assignment of proteins. J. Biomol. NMR, 30, 11-32.

Lin,G., Xu,D., Chen,Z.-Z., Jiang,T. and Xu,Y. (2002) A branch-and-bound algorithm for assignment of protein backbone NMR peaks. In First IEEE Bioinformatics Conference, pp. 165-174.

Marin,A. et al. (2004) From NMR chemical shifts to amino acid types: Investigation of the predictive power carried by nuclei. J. Biomol. NMR, 30, 47-60.

Montelione,G.T. et al. (2000) Protein NMR spectroscopy in structural genomics. Nat. Struct. Biol., 7(Suppl), 982-985.

Moseley,H.N.B. and Montelione,G.T. (1999) Automated analysis of NMR assignments and structures for proteins. Current Opinions in Structural Biol., 9, 635-642.

Seavey,B.R. et al. (1991) A relational database for sequence-specific protein NMR data. J. Biomol. NMR, 1, 217-236.

UCL/LICR (2005) The Ubiquitin NMR Resource Page. University College London/ Ludwig Institue for Cancer Research Joint NMR Laboratory.

Vitek,O. et al. (2004) Model-based assignment and inference of protein backbone nuclear magnetic resonances. Statistical Applications in Genetics and Molecular Biology, 3, 1-33.

Zhang,H. et al. (2003) A database of uniformly referenced protein chemical shifts. J. Biomol. NMR, 25, 173-195.

Zimmerman,D. et al. (1997) Automated analysis of protein NMR assignments using methods from artificial intelligence. J. Mol. Biol., 269, 592-610. 